\title{
Mortality and Recovery of Renal Function in Acute Kidney Injury Patients Treated with Prolonged Intermittent Hemodialysis Sessions Lasting 10 versus 6 Hours: Results of a Randomized Clinical Trial
}

\author{
Bianca Ballarin Albino (D), ${ }^{1}$ Mariele Gobo-Oliveira, ${ }^{1}$ \\ André Luís Balbi $\mathbb{D}^{1}{ }^{1}$ and Daniela Ponce $\mathbb{D}^{1,2}$ \\ ${ }^{1}$ Botucatu School of Medicine, University of São Paulo State (UNESP), Brazil \\ ${ }^{2}$ Course of Medicine, University of São Paulo (USP), Bauru, Brazil \\ Correspondence should be addressed to Daniela Ponce; dponce@fmb.unesp.br
}

Received 8 April 2018; Revised 26 June 2018; Accepted 25 July 2018; Published 13 August 2018

Academic Editor: Laszlo Rosivall

Copyright (C) 2018 Bianca Ballarin Albino et al. This is an open access article distributed under the Creative Commons Attribution License, which permits unrestricted use, distribution, and reproduction in any medium, provided the original work is properly cited.

\begin{abstract}
Purpose. This trial aimed to compare mortality and recovery of renal function in acute kidney injury (AKI) patients treated with different durations of prolonged hemodialysis (PHD) sessions (6h versus $10 \mathrm{~h}$ ). Methodology. We included patients with sepsisassociated AKI, $>18$ years, who are in use of a norepinephrine (lower than $0.7 \mathrm{ucg} / \mathrm{kg} / \mathrm{min}$ ). Results. One hundred and ninety-four patients were treated with 531 sessions of PHD (G1=104 and G2=90 patients). The two groups were similar in age and SOFA. There was no significant difference in hypotension, hypokalemia, and anticoagulation during PHD sessions. The two groups showed differences in filter clotting, hypophosphatemia, and treatment discontinuation $(12.3$ versus $23.1 \%, \mathrm{p}=0.002 ; 15.5$ versus $25.8 \%$, $\mathrm{p}=0.005$; and 7.9 versus $15.6 \%, \mathrm{p}=0.008$, respectively). There was no difference in fluid balance (FB) before and after PHD sessions. Death and complete recovery of renal function were similar ( 81.3 versus $82.2 \%, p=0.87$ and 21 versus $31.2 \%$, $p=0.7$, respectively). At logistic regression, the positive FB before and after dialysis was identified as risk factor for death, while volume overload after three PHD sessions and predialysis creatinine were negatively associated with recovery of renal function in 28 days. Conclusion. There was no difference in the mortality and recovery of renal function of AKI patients submitted to different durations of PHD and sessions lasting $10 \mathrm{~h}$ presented higher filter clotting, hypophosphatemia, and treatment discontinuation. ISRCTN Registry number is ISRCTN33774458.
\end{abstract}

\section{Introduction}

Acute kidney injury (AKI) is a complex and frequent syndrome in septic patients admitted to intensive care units (34\%). Despite the reduction of its mortality rate in recent years, it still remains high, reaching $62 \%$ in critical patients and $80 \%$ in those who require dialysis [1-5].

Peritoneal dialysis (PD) and hemodialysis (HD) are options for acute kidney injury support. Depending on their duration and flow of blood and dialysate they can be classified as conventional intermittent hemodialysis (IHD), prolonged intermittent hemodialysis (PHD), and continuous renal replacement therapy (CRRT) [6, 7]. So far there has been no evidence of one method being superior to the others.
PHD consists of a hybrid method with IHD characteristics, such as the use of machines and filters similar to those used in the treatment of chronic dialysis patients. It also has CRRT characteristics, such as smaller flow of blood and dialysate, between $70-250 \mathrm{ml} / \mathrm{min}$ and $70-300 \mathrm{ml} / \mathrm{min}$, respectively [8-10]. The duration of PHD therapy varies between 6 and 18 hours [7,9-11]. This method is considered to be as effective as conventional IHD and CRRT in regard to metabolic and volume control. It also has advantages when compared to CRRT, such as a lower cost, a reduced need for anticoagulation, and time optimization, with the possibility of having the patient undergo exams and procedures away from the ICU [12-14]. 
At the moment the literature does not include any studies that have evaluated and compared mortality rates and recovery of renal function in patients with AKI treated with PHD sessions of different durations.

This clinical trial was designed to evaluate and compare the recovery of renal function, mortality, and intra- and postdialysis complications in critically ill AKI patients undergoing PHD sessions lasting 6 or $10 \mathrm{~h}$. We hypothesized that PHD sessions lasting 10 hours would cause less hypotension than PHD sessions lasting 6 hours, leading to faster partial recovery of renal function.

\section{Methods}

2.1. Studied Population. This is a randomized clinical trial conducted from January 2012 to March 2016 in patients enrolled in the Brazilian University Hospital. The protocol was approved by the Institutional Ethical Committee (CAAE Protocol: 28146714.6.0000.5411). Written informed consent was obtained from patients or their next of kin. The study also was registered in the ISRCTN Registry under the number ISRCTN33774458.

Patients were eligible for enrolment if they were 18 years of age or older, with AKI associated with sepsis and on a noradrenaline dose lower than $0.7 \mu \mathrm{g} / \mathrm{kg} / \mathrm{min}$. AKI was defined according to of KDIGO [15]. Exclusion criteria were chronic kidney disease stages 4 and 5, previous chronic dialysis, kidney transplantation, patients previously treated with any acute dialysis during ICU stay, pregnancy, and taking noradrenaline dose higher than $0.7 \mu \mathrm{g} / \mathrm{kg} / \mathrm{min}$. These last patients were excluded because they could not tolerate actual ultrafiltration (UF) of $300-500 \mathrm{~mL} / \mathrm{h}$ and because of that, they were treated with CRRT.

2.2. Criteria for Initiating and Stopping PHD. The indications for dialysis were uraemic symptoms, BUN level $>100 \mathrm{mg} / \mathrm{dL}$ (azotaemia), volume overload, electrolyte imbalance (potassium $>6 \mathrm{mEq} / \mathrm{L}$ after clinical treatment), or acid-base refractory disturbances (bicarbonate $<10 \mathrm{mEq} / \mathrm{L}$ after reposition).

A patient was considered for enrolment if the judgment of the treating nephrologists was that he or she required dialysis and the mean arterial blood pressure (BP) was higher than $80 \mathrm{mmHg}$, with a noradrenaline dose lower than $0.7 \mu \mathrm{g} / \mathrm{kg} / \mathrm{min}$ in the 8 hours preceding randomization.

The patients were randomly assigned to two groups, according to the treatment duration prescribed. The randomization was computerized by the randomization.com website. hours

Group 1: Patients treated with PHD sessions lasting 6 hours

Group 2: Patients treated with PHD sessions lasting 10

The hemodialysis sessions were accompanied by the same research team until the patient's final clinical outcome (recovery of renal function, change of dialysis method, or death).

After diagnosing the sepsis-associated AKI and indicating PHD for dialysis, the central venous catheter was implanted to initiate treatment. The PHD session lasted 6 or 10 hours according to randomization and, for practical reasons, it was decided that PHD would be carried out 6 days a week (Monday-Saturday).

We used proportion machines (Fresenius 4008) and capillary polysulfone membranes (surface areas of 1.0 and $1.2 \mathrm{~m}^{2}$ ) for the sessions.

The PHD sessions were performed with blood and dialysate flows of 200 and $300 \mathrm{~mL} / \mathrm{min}$, respectively. For Group 1 we used FX 80 capillary dialyzers, while for Group 2 we used FX 60 capillary dialyzers in order to minimize the difference between the dialysis doses provided. During the sessions, the patients were anticoagulated with a 50 to $100 \mathrm{IU} / \mathrm{kg}$ bolus dose of heparin, and then with 500 to $1000 \mathrm{IU} /$ hour in the following hours. In cases of contraindication to anticoagulation, the system was washed with $50 \mathrm{ml}$ of $0.9 \%$ sodium chloride every 30 minutes, throughout the entire procedure. The concentrations of bicarbonate (26$36 \mathrm{mEq} / \mathrm{L})$, potassium (1-3 mEq/L), sodium (140-145 mEq/L), and calcium ( 2.5 to $3.5 \mathrm{mEq} / \mathrm{L}$ ) of the dialysis bath were adjusted according to the exams and individual needs of the patients. The ultrafiltration rate (UF) did not exceed $500 \mathrm{ml} /$ hour and the bath temperature ranged from 35 to $35.5^{\circ} \mathrm{C}$.

We evaluated hypotension and filter clotting as intradialytic complications. Hypotension was defined as systolic blood pressure (BP) below $90 \mathrm{mmHg}$, or as a sudden BP drop of $20 \mathrm{mmHg}$. Filter clotting was defined as the presence of blood clots in the circuit, composed of a dialyzer and lines, precluding the continuation of therapy. As preventive measures to hypotension we used UF rates not exceeding $500 \mathrm{ml} / \mathrm{h}$, dialysate temperature between 35 and $35.5^{\circ} \mathrm{C}$, and high dialysate sodium concentration (140-145 mEq/L). When we observed the presence of thrombi and blood clots in the system, we conducted saline flushes or administered an extra dose of heparin to prevent coagulation, according to medical prescription. As postdialysis complications we evaluated the presence of hypokalemia and hypophosphatemia, characterized by serum levels below $3.5 \mathrm{mEq} / \mathrm{l}$ and $3.5 \mathrm{mg} / \mathrm{dl}$, respectively.

We evaluated the outcomes of death and recovery of renal function in 28 days. The recovery of renal function was assessed using the relationship between the reference creatinine level and the creatinine level at 28 days after hospital discharge (reference $\mathrm{Cr} / \mathrm{Cr}$ at 28 days) and classified the recovery as complete if above 0.9 , partial if between 0.5 and 0.9 , and as nonrecovery if below 0.5. [16]

We collected the following clinical data: name, gender, age, race, presence of comorbidities (diabetes, chronic kidney disease, and hypertension), primary diagnosis, sepsis etiology, and AKI specific prognostic score (ATN-ISS) [17], Sequential Organ Failure Assessment (SOFA) [18], date of hospitalization, PHD starting date, concentration of vasoactive drug before and after PHD. We quantified urea, creatinine, sodium, potassium, phosphorus, calcium, and venous gas every day, before and after HD. Posttreatment BUN levels were measured by the slow flow method (with blood pump speed reduced to $50 \mathrm{~mL} / \mathrm{min}$ ). Blood samples were obtained from the arterial sampling port before the blood reached the dialyzer. HD adequacy was determined by using urea 
kinetic modelling based on Kt/V [19]. The delivered dose was determined by the single-pool Kt/V value, corrected for actual UF but not for the reappearance of urea nitrogen [19].

As dialytic therapy data we evaluated the number of sessions performed and of filters used, blood and dialysate flows, volume of UF prescribed and obtained, urea removal rate, BP every 30 minutes, and the presence of hypotension and filter clotting as previously defined. To solve hypotension during PHD, we applied protocols which included saline infusion, decreasing or discontinuing UF and increasing the vasoactive drug, according to the clinical and volume conditions of the patient. Therapy was interrupted when, despite taking these measures, hemodynamic instability persisted and presented risks to the patient.

Dialysis was interrupted when there was partial recovery of renal function (dialysis-independent) defined as restoration of urine output higher than $1000 \mathrm{~mL} / 24 \mathrm{~h}$ associated with a progressive fall in serum values for creatinine $(<4 \mathrm{mg} / 100 \mathrm{~mL})$ and BUN $(<50 \mathrm{mg} / \mathrm{dL})$, a need to change dialysis method because of infectious, mechanical, or haemodynamic complications, more than 30 days of follow-up, or death.

2.3. Statistical Analysis. Considering alpha error as 5\%, beta error as $20 \%$, statistical power of the test as $80 \%$, and detecting a $15 \%$ mortality rate difference between groups, the sample size for each group was calculated as 94 patients.

We described the variables with normal distribution as mean value \pm standard deviation and the variables with nonnormal distribution as mean value and interquartile range.

We performed comparisons of the continuous variables between the two groups using Student's t-test for data with normal distribution and the Mann-Whitney test for nonnormal data. For the comparative analysis of categorical variables we used the Chi-squared tests. For comparing variables by session between the groups we used the mixed model of repeated measures over time with an adjustment for Tukey's test. We used univariate and multivariate linear regression for the association of risk factors for death and nonrecovery of renal function in 28 days.

We considered a $5 \%$ significance level in all of the tests performed.

For data analysis we used the SAS program for Windows, version 9.2 (developed in 2009, in Cary, North Carolina, USA).

\section{Results}

A hundred and ninety-four patients with sepsis-associated AKI received 531 PHD sessions. The mean age was $60.8 \pm 14.9$ years, and $69.5 \%$ of the patients were male. The main infection focus was pulmonary (41.2\%) and hypertension was the most prevalent of the comorbidities (52.5\%). The specific prognosis index for AKI, the ATN-ISS, was $0.77 \pm 0.1$ and the mean SOFA score was $14.2 \pm 2.9$.

G1 was composed of 104 patients, treated for 276 sessions. G2 was composed of 90 patients, treated for 255 sessions. When comparing clinical characteristics, both groups were similar in relation to the predominance of males $(70.1 \%$ in G1 and $68.8 \%$ in $\mathrm{G} 2, \mathrm{p}=0.84$ ), mean age of $61.4 \pm 14.4$ years in G1 and $60.5 \pm 15.5$ years in G2 ( $\mathrm{p}=0.55)$, presence of comorbidities such as hypertension and diabetes (55.7\% versus $48.8 \%, \mathrm{p}=0.33$ and $27.8 \%$ versus $26.6 \%, \mathrm{p}=0.84$, respectively), specific prognosis for ATN (ATN-ISS), and SOFA (0.76 \pm 0.1 versus $0.77 \pm 0.2, p=0.87$ and $14.1 \pm 3$ versus $14.4 \pm 2.9$ $\mathrm{p}=0.47$, respectively). In Group 1, pulmonary infection focus was prevalent $(41.3 \%)$, while in Group 2, the main focus was abdominal $(42.2 \%, \mathrm{p}=0.07)$. Most patients were on mechanical ventilation, $94.2 \%$ in Group 1, compared to $93.3 \%$ in Group $2(p=0.96)$. The groups were similar regarding initial and final doses of vasoactive drug $(0.56$ versus $0.55, \mathrm{p}$ $=0.97$ and 0.69 versus $0.7, \mathrm{p}=0.91$, respectively) as shown in Table 1.

Table 2 shows the presence of dialysis complications during hemodialysis sessions, in general and divided by groups. The main complication among them was hypotension (50\%), followed by filter clotting, hypophosphatemia, and hypokalemia, which happened in $17.5 \%, 20.5 \%$, and $11.2 \%$ of the sessions, respectively. There was a significant difference between the groups in relation to filter clotting (12.3 versus $23.1 \%, \mathrm{p}=0.002$ ), hypophosphatemia ( 15.5 versus $25.8 \%, \mathrm{p}$ $=0.005)$, and hemodialysis session interruption due to the presence of persistent hypotension after refractory measures ( 7.9 versus $15.6 \%, p=0.008$ ). Both groups were similar in relation to hypotension ( 46.7 versus $53.7 \%, \mathrm{p}=0.13$ ), hypokalemia (11.5 versus $10.9 \%, \mathrm{p}=0.93$ ), and the use of anticoagulation in the PHD sessions (45.2 versus $36.8 \%$, $\mathrm{p}=$ $0.06)$.

The main outcome was death, present in $81.7 \%$ of the general population. Recovery of renal function (RF) was assessed among the survivors: $25.7 \%$ presented complete recovery, $68.5 \%$ presented partial recovery, and $5.7 \%$ presented nonrecovery. There was no difference between the groups in relation to outcomes (death: 81.3 versus $82.2 \%, \mathrm{p}=$ 0.87 ; complete recovery of FR: 21 versus $31.2 \%, \mathrm{p}=0.7$; partial recovery of RF: 68.4 versus $68.7 \%, p=0.7$; nonrecovery of RF: 10.5 versus $0 \%, \mathrm{p}=0.48$ ), as shown in Table 3 .

The metabolic and volume controls of the AKI patients treated with PHD sessions lasting 6 and 10 hours were assessed after the first three sessions and are presented in Table 4. The target metabolic and volume control was reached in both groups we studied with urea lower than $120 \mathrm{mg} / \mathrm{dl}$, $\mathrm{pH}$ above 7.3, minimum weekly Kt/v of 3.9, and cumulative fluid balance close to zero after 3 sessions. The groups showed differences in the values of urea reduction ratio (URR) on the first and third sessions (S1: $0.6 \pm 0.1$ versus $0.68 \pm 0.1$; $\mathrm{p}<$ 0.001 , S3: $0.56 \pm 0.1$ versus $0.62 \pm 0.1 ; p=0.03$ ), being higher in G2. Potassium and phosphorous serum values were $(4.3 \pm 0.8$ versus $3.9 \pm 0.6, p=0.04 ; 5.8 \pm 2.2$ versus $4.1 \pm 1.6, p=0.009$, respectively), being lower in G2. The rate of ultrafiltration (UF) prescribed in the first three sessions was (S1: $2064 \pm 927$ versus $2580 \pm 1000, \mathrm{p}=0.0002$; S2: $2262 \pm 852$ versus $2626 \pm$ $1123, \mathrm{p}=0.02$, and $\mathrm{S} 3: 2217 \pm 755$ versus $2656 \pm 1004, \mathrm{p}=0.03$ )

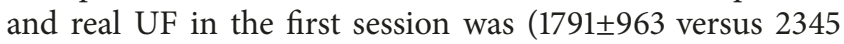
$\pm 1017, p=0.0006)$, which were higher in G2. However, the UF in $\mathrm{ml} / \mathrm{h}$ was lower in G2 in all sessions we studied. The other parameters assessed were similar between the groups. 
TABLE 1: Clinical and laboratory characteristics of patients with AKI treated with PHD.

\begin{tabular}{|c|c|c|c|c|}
\hline Parameters & General $(n=194)$ & G1 $(n=104)$ & G2 $(n=90)$ & p value \\
\hline Age (in years) & $60.8 \pm 14.9$ & $61.4 \pm 14.4$ & $60.2 \pm 15.5$ & 0.55 \\
\hline Males, n (\%) & $135(69.5)$ & $73(70.1)$ & $62(68.8)$ & 0.84 \\
\hline Weight & $74.8 \pm 22.5$ & $74.1 \pm 24$ & $75.6 \pm 20.8$ & 0.63 \\
\hline \multicolumn{5}{|l|}{ Infectious focus n (\%) } \\
\hline Pulmonary & $80(41.2)$ & $43(41.3)$ & $37(41.2)$ & 0.97 \\
\hline Abdominal & $69(35.5)$ & $31(29.8)$ & $38(42.2)$ & 0.07 \\
\hline \multicolumn{5}{|l|}{ Comorbidities, n (\%) } \\
\hline (i) $\mathrm{SAH}$ & $102(52.5)$ & $58(55.7)$ & $44(48.8)$ & 0.33 \\
\hline (ii) $\mathrm{DM}$ & $53(27.2)$ & $29(27.8)$ & $24(26.6)$ & 0.84 \\
\hline (iii) $\mathrm{CKD}$ & $19(9.7)$ & $12(11.5)$ & $7(7.7)$ & 0.37 \\
\hline ATN-ISS & $0.77 \pm 0.1$ & $0.76 \pm 0.1$ & $0.77 \pm 0.2$ & 0.87 \\
\hline SOFA & $14.2 \pm 2.9$ & $14.1 \pm 3$ & $14,4 \pm 2,9$ & 0.47 \\
\hline Pre-dialysis FB (l) & $3.36 \pm 1.8$ & $3.27 \pm 1.9$ & $3.47 \pm 1.7$ & 0.47 \\
\hline $\mathrm{Ur}(\mathrm{mg} / \mathrm{dl})$ & $155.3 \pm 105.7$ & $158.6 \pm 59.5$ & $151.6 \pm 141.2$ & 0.64 \\
\hline $\mathrm{Cr}(\mathrm{mg} / \mathrm{dl})$ & $3.7 \pm 1.5$ & $3.7 \pm 1.4$ & $3.6 \pm 1.7$ & 0.64 \\
\hline $\mathrm{K}(\mathrm{mEq} / \mathrm{L})$ & $4.7 \pm 1$ & $4.7 \pm 1$ & $4.8 \pm 1$ & 0.48 \\
\hline $\operatorname{Bic}(\mathrm{mEq} / \mathrm{L})$ & $19.1 \pm 4.6$ & $18.8 \pm 4.7$ & $19.5 \pm 4.6$ & 0.39 \\
\hline Mechanical Ventilation & $182(93.8)$ & $98(94.2)$ & $84(93.3)$ & 0.96 \\
\hline Initial vasoactive drug dose & $0.55 \pm 0.18$ & $0.55 \pm 0.17$ & $0.56 \pm 0.19$ & 0.97 \\
\hline Final vasoactive drug dose & $0.69 \pm 0.19$ & $0.69 \pm 0.16$ & $0.70 \pm 0.20$ & 0.91 \\
\hline
\end{tabular}

Values are presented in frequency, mean values and standard deviation, median, and proportions.

AKI: acute renal injury, PHD: prolonged hemodialysis, SAH: systemic arterial hypertension, DM: diabetes mellitus, CKD: chronic kidney disease, ATN-ISS: acute tubular necrosis individual severity score, SOFA: sequential organ failure assessment score, FB: fluid balance, Ur: urea, Cr: creatinine, K: potassium, Bic: bicarbonate, and UF: ultrafiltration.

TABLE 2: Dialysis complications by PHD sessions according to the groups studied.

\begin{tabular}{lcccc}
\hline Complications & $\begin{array}{c}\text { General } \\
(\mathbf{n}=\mathbf{5 3 1})\end{array}$ & $\begin{array}{c}\text { G1 } \\
(\mathbf{n = 2 7 6 )}\end{array}$ & $\begin{array}{c}\text { G2 } \\
(\mathbf{n = 2 5 5 )}\end{array}$ & $\mathbf{p}$ value \\
\hline Hypotension, n (\%) & $266(50)$ & $129(46.7)$ & $137(53.7)$ \\
Filter clotting, n (\%) & $93(17.5)$ & $34(12.3)$ & $59(23.1)$ \\
Hypokalemia, n (\%) & $60(11.2)$ & $32(11.5)$ & $28(10.9)$ & 0.13 \\
Hypophosphataemia, n (\%) & $109(20.5)$ & $43(15.5)$ & $66(25.8)$ & 0.002 \\
Use of anticoagulation, n (\%) & $219(41.2)$ & $125(45.2)$ & $94(36.8)$ & 0.93 \\
Treatment discontinuation, n (\%) & $62(11.6)$ & $22(7.9)$ & $40(15.6)$ & 0.005 \\
\hline
\end{tabular}

Values are presented in proportions.

PHD: prolonged hemodialysis.

Fluid balance was assessed before the first and third sessions of $\mathrm{PHD}$, and there was no difference between the groups ( $\mathrm{S} 1$ : $3.27 \pm 1.9$ versus $3.47 \pm 1.7, p=0.47$ and $S 3: 1.33 \pm 2.6$ versus $0.47 \pm 2.4, \mathrm{p}=0.09)$.

A logistic regression was conducted for the death outcomes and the variables of weight, SOFA, fluid balance before and after 3 sessions, presession potassium, and the presence of hypokalemia were identified as risk factors. After the multivariate analysis, the association remained only for fluid balance before and after 3 sessions. This data is included in Tables 5 and 6.

Similarly, the logistic regression was carried out for the recovery of renal function in 28 days, and the values of fluid balance before and after 3 sessions and creatinine presession presented significant negative association, which remained after the multivariate analysis. This data is shown in Tables 7 and 8 .

\section{Discussion}

This clinical trial study aimed to assess and compare the mortality rate and recovery of renal function in critical patients with AKI, treated with PHD sessions of different durations (6 and 10 hours). There are very few studies on PHD in the literature and, until now, none of them compared the clinical evolution of patients in PHD of different durations.

Hypotension was the most common complication and occurred in $50 \%$ of the sessions, despite the use of precautionary measures, such as low dialysate temperature, high sodium concentrations, and UF rate not exceeding $500 \mathrm{ml} / \mathrm{h}$. Similar 
TABle 3: Main outcomes of patients with AKI treated with PHD according to the duration of the PHD sessions.

\begin{tabular}{lcccc}
\hline Outcomes & $\begin{array}{c}\text { General } \\
(\mathbf{n}=\mathbf{1 9 4})\end{array}$ & $\begin{array}{c}\text { G1 } \\
(\mathbf{n}=\mathbf{1 0 4})\end{array}$ & $\begin{array}{c}\text { G2 } \\
(\mathbf{n}=\mathbf{9 0})\end{array}$ & $\mathbf{p}$ value \\
\hline Death, n (\%) & $157(81.7)$ & $83(81.3)$ & $4(82.2)$ & 0.87 \\
Complete recovery of RF, n (\%) & $9(25.7)$ & $13(21)$ & $11(68.7)$ & 0.7 \\
Partial recovery of RN, n (\%) & $24(68.5)$ & $2(10.5)$ & 0 & 0.7 \\
Non-recovery of RF, n (\%) & $2(5.7)$ & & 0.48 \\
\hline
\end{tabular}

Values are presented in proportions.

AKI: acute renal injury, PHD: prolonged hemodialysis, and RF: renal function.

results were reported by Fieghen et al. [20], Ponce et al. [21], And Albino et al. [22].

However, there was no difference between the groups treated in our study. The longest session duration time in Group 2 resulted in a lower ultrafiltration rate (ml/hour), though it did not prevent and/or improve the frequency of hypotension. The dose of the vasoactive substance was higher at the end of the PHD sessions, when compared to the dose at the beginning, in an attempt to keep arterial pressure steady during therapy, and it was similar between the groups.

The second most frequent dialysis complication in our study was filter clotting, which occurred in $11.2 \%$ sessions, similar to the data reported in the literature $[3,12,21,22]$. The use of anticoagulation in the PHD sessions was carried out according to the comorbidities and bleeding risk related to the patient. Anticoagulation occurred in $41.2 \%$ of the sessions and there was no difference between the groups.

The prevalence of filter clotting was different between the groups as was treatment discontinuation. These facts may be related to the longer duration of treatment of Group 2 patients, making them more susceptible to the persistence of intradialytic complications.

Hypokalemia and hypophosphatemia are postdialysis complications that are rarely addressed in the previous studies conducted on PHD, which complicates the analysis and comparison of the results obtained in our study. Marshall et al. [23] analyzed 145 PHD sessions in 37 patients and found hypokalemia and hypophosphatemia in 7 (4.8\%) and $18(12.4 \%)$ occurrences, respectively, and these results are similar to those found in our study. Similarly, Palevsky et al. [24] found a prevalence of hypophosphatemia in $12.4 \%$ of the patients treated with PHD in their ATN study.

There was a difference between the groups we studied in relation to hypophosphatemia, probably associated with the longer therapy duration and consequently greater removal of solutes, and the groups were similar in relation to hypokalemia.

The target metabolic and volume control was reached in both groups we studied. However, there was difference between the groups regarding the URR and $\mathrm{Kt} / \mathrm{V}$, which were higher in group 2. Despite the use of capillaries with a smaller area for Group 2, due to the 10-hour treatment duration, these patients received a slightly higher Kt/v.

Although the Kt/V was higher in Group 2, the mortality rate was similar between the groups, in accordance with previous studies that showed that more intensive dialysis is not associated with better outcomes $[25,26]$.

The ultrafiltration value was prescribed according to the fluid balance of the patient, and it ranged between 1500 and $3000 \mathrm{ml}$ per session. There was a difference between the groups. Group 2 had higher prescribed and actual UF. However, the fluid balance was similar between the studied populations after 3 sessions of therapy. There was no difference in cumulative fluid balance before and after 3 dialysis sessions between the groups.

Death occurred in $81.7 \%$ of the general population and there was no difference between the groups. The mortality rate we found was higher than those reported in previous studies carried out in European countries and in North America, such as in a study conducted in Toronto by Kitchlu et al., who observed death in $54 \%$ of patients treated with PHD [12]. Our mortality rate, however, was similar to those observed in critical patients with AKI in developing countries. In Brazilian studies, mortality of AKI patients that underwent dialysis ranged from 67 to $85 \%[3,22,27,28]$. This data is similar to that described by George et al., who performed a study in India and obtained mortality over 75\% in patients with AKI [29].

Considering we included patients with sepsis-associated AKI and elevated prognostic indexes (ATN-ISS and SOFA of $0.77 \pm 0.1$ and $14.2 \pm 2.9$, respectively), the patients studied were in severe conditions, which justifies the unfavorable outcomes. It is important to emphasize that we only evaluated septic patients, which was not made in other studies.

We identified cumulative fluid balance before dialysis and after 3 sessions of PHD as the only death-associated factor. These results are in agreement with previous studies that reported low urine output, fluid overload, and sepsis are associated with worse prognostic of AKI patients [21, $26,30,31$. Clinical data show that positive fluid balance and oliguria can contribute negatively to prognosis lung, leading to increased time of invasive mechanical ventilation, durations of hospitalization, and mortality [30, 31].

In our study, we could not identify dialysis dose as a risk factor for death, in agreement with Palevsky et al. [25] and Bellomo et al. [26] in the trials ATN and RENAL, respectively.

Recovery of renal function was assessed among the survivors: $25.7 \%$ had complete recovery and $68.5 \%$ had partial recovery. Similar results reported that a quarter of the patients obtained complete recovery of renal function after 30 days. In our study, the predialysis creatinine value and fluid balance 


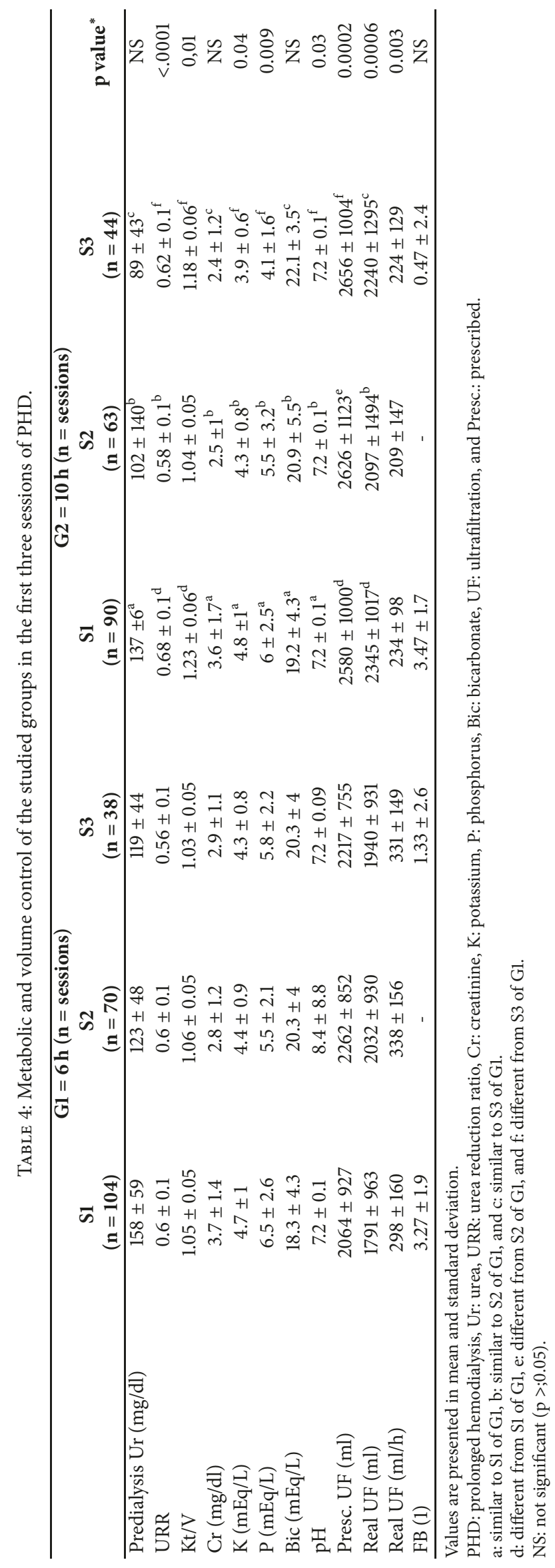


TABLE 5: Univariate logistic regression of clinical and laboratory characteristics, and dialysis complications associated with the death of patients with AKI treated with PHD.

\begin{tabular}{|c|c|c|c|}
\hline Parameter & OR & Confidence Interval & p value \\
\hline Age & 1.01 & $0.99-1.03$ & 0.24 \\
\hline Gender & 0.60 & $0.28-1.28$ & 0.19 \\
\hline Weight & 1.01 & $1.00-1.03$ & 0.04 \\
\hline Infectious focus & 0.95 & $0.33-2.70$ & 0.87 \\
\hline SAH & 0.84 & $0.40-1.75$ & 0.64 \\
\hline $\mathrm{DM}$ & 0.73 & $0.31-1.74$ & 0.48 \\
\hline CKD & 0.22 & $0.02-1.76$ & 0.15 \\
\hline ATN-ISS & 0.94 & $0.11-7.98$ & 0.95 \\
\hline SOFA & 1.22 & $1.06-1.40$ & 0.004 \\
\hline Pre FB & 1.47 & $1.12-1.93$ & 0.004 \\
\hline Post FB & 1.38 & $1.11-1.72$ & 0.003 \\
\hline Pre Ur & 0.99 & $0.99-1.0$ & 0.15 \\
\hline Post Ur & 1.00 & $0.99-1.01$ & 0.67 \\
\hline Pre Cr & 1.01 & $0.80-1.28$ & 0.88 \\
\hline CR post & 0.94 & $0.60-1.47$ & 0.78 \\
\hline Pre K & 1.79 & $1.18-2.73$ & 0.006 \\
\hline Post K & 1.82 & $0.82-4.00$ & 0.13 \\
\hline Pre Bic & 0.93 & $0.85-1.01$ & 0.11 \\
\hline Post Bic & 0.90 & $0.75-1.08$ & 0.27 \\
\hline Pre UF & 0.93 & $0.78-1.12$ & 0.49 \\
\hline Post UF & 0.86 & $0.67-1.12$ & 0.28 \\
\hline RF outcome & 1.0 & $<0,001->999,999$ & 0.85 \\
\hline Hypotension & 0.50 & $0.23-1.09$ & 0.008 \\
\hline Coagulation & 0.76 & $0.34-1.70$ & 0.5 \\
\hline Hypokalemia & 3.7 & $1.62-8.83$ & 0.002 \\
\hline Hypophosphataemia & 1.48 & $0.66-3.31$ & 0.33 \\
\hline
\end{tabular}

Or: odds ratio.

AKI: acute renal injury, PHD: prolonged hemodialysis, SAH: systemic arterial hypertension, DM: diabetes mellitus, CKD: chronic kidney disease, ATN-ISS: acute tubular necrosis individual severity score, SOFA: sequential organ failure assessment score, FB: fluid balance, Ur: urea, Cr: creatinine, K: potassium, Bic: bicarbonate, UF: ultrafiltration, and RF: renal function. Pre $=1$ st session, post $=3$ rd session of PHD.

TABLE 6: Multivariate logistic regression of clinical and laboratory characteristics and dialysis complications associated with the death of patients with AKI treated with PHD.

\begin{tabular}{llcr}
\hline Parameter & OR & Confidence Interval & p value \\
\hline Weight & 1.01 & $0.98-1.04$ & 0.51 \\
SOFA & 1.08 & $0.34-1.38$ & 0.52 \\
Pre FB & 1.60 & $1.04-2.47$ & 0.03 \\
Post FB & 1.54 & $1.16-2.04$ & 0.002 \\
Hypokalemia & 1.69 & $0.39-7.22$ & 0.47 \\
\hline
\end{tabular}

Or: odds ratio.

AKI: acute kidney injury, HDP: prolonged hemodialysis, SOFA: sequential organ failure assessment score, and FB: fluid balance.

after 3 sessions were identified as risk factors for the recovery of renal function. Hamzić-Mehmedbašić et al. identified that the female gender, comorbidities, and sepsis were risk factors for a worse evolution of renal function [32].

Our study presents several limitations, such as the small number of studied patients and its execution in a single center. Due to the different duration of treatment between the groups, we were not able to perform the randomization blindly. The assessment of long-term survival was also not performed. Despite these limitations, this was the first study to assess the clinical evolution of patients with AKI treated with different durations of PHD [33].

In conclusion, our results show that mortality and recovery of renal function are similar between the groups treated with PHD lasting 6 and $10 \mathrm{~h}$. However, Group 2 showed higher incidence of dialysis complications, such as filter clotting and hypophosphatemia, probably related to the 
TABLE 7: Univariate logistic regression of clinical and laboratory characteristics and dialysis complications associated with the recovery of renal function in patients with AKI treated with PHD.

\begin{tabular}{|c|c|c|c|}
\hline Parameter & OR & Confidence Interval & p value \\
\hline Age & 1.01 & $0.99-1.03$ & 0.17 \\
\hline Gender & 0.74 & $0.37-1.50$ & 0.41 \\
\hline Weight & 0.99 & $0.97-1.00$ & 0.27 \\
\hline \multicolumn{4}{|l|}{ Infectious focus } \\
\hline $\mathrm{SAH}$ & 0.66 & $0.34-1.27$ & 0.21 \\
\hline $\mathrm{DM}$ & 0.98 & $0.48-1.97$ & 0.95 \\
\hline CKD & 1.9 & $0.52-7.2$ & 0.32 \\
\hline ATN-ISS & 1.17 & $0.15-9.18$ & 0.87 \\
\hline SOFA & 0.92 & $0.81-1.04$ & 0.19 \\
\hline Pre FB & 0.93 & $0.78-1.10$ & 0.41 \\
\hline Post FB & 0.83 & $0.69-0.99$ & 0.04 \\
\hline Pre Ur & 1.00 & $0.99-1.00$ & 0.71 \\
\hline Post Ur & 0.99 & $0.98-1.00$ & 0.65 \\
\hline Pre $\mathrm{Cr}$ & 0.88 & $0.57-0.96$ & 0.03 \\
\hline Post $\mathrm{Cr}$ & 1.00 & $0.97-1.03$ & 0.57 \\
\hline Pre K & 0.77 & $0.56-1.05$ & 0.10 \\
\hline Post K & 0.68 & $0.34-1.38$ & 0,29 \\
\hline Pre Bic & 1.07 & $0.99-1.15$ & 0.08 \\
\hline Post Bic & 1.00 & $0.85-1.18$ & 0.92 \\
\hline Pre UF & 1.09 & $0.94-1.27$ & 0,24 \\
\hline Post UF & 0.94 & $0.72-1.23$ & 0,67 \\
\hline Hypotension & 1.8 & $0.92-3.67$ & 0,08 \\
\hline Coagulation & 0.86 & $0.44-1.69$ & 0.67 \\
\hline Hypokalemia & 0.74 & $0.33-1.67$ & 0.47 \\
\hline Hypophosphataemia & 1.10 & $0.53-2.29$ & 0.78 \\
\hline
\end{tabular}

OR: odds ratio.

AKI: acute renal injury, PHD: prolonged hemodialysis, SAH: systemic arterial hypertension, DM: diabetes mellitus, CKD: chronic kidney disease, ATN-ISS: acute tubular necrosis individual severity score, SOFA: sequential organ failure assessment score, FB: fluid balance, Ur: urea, Cr: creatinine, K: potassium, Bic: bicarbonate, UF: ultrafiltration, and RF: renal function. Pre $=1$ st session, post $=3$ rd session of PHD.

TABLE 8: Multivariate logistic regression of clinical and laboratory characteristics and dialysis complications associated with the recovery of renal function in patients with AKI treated with PHD.

\begin{tabular}{lccc}
\hline Parameter & OR & Confidence Interval & $p$ value \\
\hline SOFA & 0.86 & $0.68-1.12$ & 0.48 \\
Pre FB & 0.94 & $0.72-1.08$ & 0.08 \\
Post FB & 0.98 & $0.65-0.97$ & 0.009 \\
Pre Cr & 0.82 & $0.59-0.91$ & 0.04 \\
\hline
\end{tabular}

OR: odds ratio.

AKI: acute kidney injury, HDP: prolonged hemodialysis, SOFA: sequential organ failure assessment score, FB: fluid balance, and Pre Cr: creatinine at 1st PHD session.

extended duration of therapy. Therefore, there is no benefit in treating patients with 10 -hour sessions.

Future work in this area should aim to clarify factors that inform decision-making around time of PHD modality. Larger and trial studies will need to clarify the impact of PHD on patient survival and recovery of renal function.

\section{Data Availability}

The data are available in UNESP thesis repository: http://hdl .handle.net/11449/151845.

\section{Disclosure}

All authors have agreed to the submission to the journal and that the manuscript is not currently under submission in any other journal.

\section{Conflicts of Interest}

All authors declare that there are no conflicts of interest. 


\section{References}

[1] R. L. Mehta, J. A. Kellum, S. V. Shah et al., "Acute Kidney Injury Network: report of an initiative to improve outcomes in acute kidney injury," Critical Care, vol. 11, no. 2, article R31, 2007.

[2] C. H. Chang, P. C. Fan, M. Y. Chang et al., "Acute kidney injury enhances outcome prediction ability of sequential organ failure assessment score in critically ill patients," PLoS ONE, vol. 9, no. 10, 2014.

[3] R. A. Caires, R. C. R. M. Abdulkader, V. T. Costa e Silva et al., "Sustained low-efficiency extended dialysis (SLED) with singlepass batch system in critically-ill patients with acute kidney injury (AKI)," Journal of Nephrology, vol. 29, no. 3, pp. 401-409, 2016.

[4] J. Himmelfarb and T. A. Ikizler, "Acute kidney injury: changing lexicography, definitions, and epidemiology," Kidney International, vol. 71, no. 10, pp. 971-976, 2007.

[5] S. Uchino, J. A. Kellum, R. Bellomo et al., "Acute renal failure in critically ill patients: a multinational, multicenter study," The Journal of the American Medical Association, vol. 294, no. 7, pp. 813-818, 2005.

[6] N. Pannu, S. Klarenbach, N. Wiebe, B. Manns, and M. Tonelli, "Renal replacement therapy in patients with acute renal failure: a systematic review," Journal of the American Medical Association, vol. 299, no. 7, pp. 793-805, 2008.

[7] J. T. Kielstein, M. Schiffer, and C. Hafer, "Back to the future: extended dialysis for treatment of acute kidney injury in the intensive care unit," Journal of Nephrology, vol. 23, no. 5, pp. 494-501, 2010.

[8] R. Shingarev, K. Wille, and A. Tolwani, "Management of complications in renal replacement therapy," Seminars in Dialysis, vol. 24, no. 2, pp. 164-168, 2011.

[9] W. Sulowicz and A. Radziszewski, "Pathogenesis and treatment of dialysis hypotension," Kidney International, vol. 70, no. 104, pp. S36-S39, 2006.

[10] R. A. Sherman, J. T. Daugirdas, and T. S. Ing, "Complications during hemodialysis," in Handbook of Dialysis, J. T. Daugirdas, P. G. Blake, and T. S. Ing, Eds., pp. 158-164, Lippincott Williams \& Wilkins, Philadelphia, PA, USA, 4th edition, 2007.

[11] M. Doshi and P. T. Murray, "Approach to intradialytic hypotension in intensive care unit patients with acute renal failure," Artificial Organs, vol. 27, no. 9, pp. 772-780, 2003.

[12] A. Kitchlu, N. Adhikari, K. E. Burns et al., "Outcomes of sustained low efficiency dialysis versus continuous renal replacement therapy in critically ill adults with acute kidney injury: a cohort study," BMC Nephrology, vol. 16, no. 1, 2015.

[13] L. Zhang, J. Yang, G. M. Eastwood, G. Zhu, A. Tanaka, and R. Bellomo, "Extended daily dialysis versus continuous renal replacement therapy for acute kidney injury: a meta-analysis," American Journal of Kidney Diseases, vol. 66, no. 2, pp. 322-330, 2015.

[14] M. M. Levy, R. P. Delinger, S. R. Townsend et al., "Surviving sepsis campaign: results of an international guideline-based performance improvement program targeting severe sepsis," Critical Care Medicine, vol. 38, pp. 367-374, 2010.

[15] Kidney Disease: Improving Global Outcomes (KDIGO) CKD Work Group, "KDIGO 2012 clinical practice guideline for the evaluation and management of chronic kidney disease," Kidney International Supplements, vol. 3, pp. 1-150, 2013.
[16] J.-P. Lafrance and D. R. Miller, "Acute kidney injury associates with increased long-term mortality," Journal of the American Society of Nephrology, vol. 21, no. 2, pp. 345-352, 2010.

[17] F. Liano, A. Gallego, J. Pascual et al., "Prognosis of acute tubular necrosis: an extended prospectively contrasted study," Nephron, vol. 63, no. 1, pp. 21-31, 1993.

[18] F. Lopes Ferreira, D. Peres Bota, A. Bross, C. Mélot, and J.L. Vincent, "Serial evaluation of the SOFA score to predict outcome in critically ill patients," Journal of the American Medical Association, vol. 286, no. 14, pp. 1754-1758, 2001.

[19] J. T. Daugirdas, "Physiologic principles and urea kinetic modeling," in Handbook of Dialysis, J. T. Daugirdas, P. G. Blake, and T. S. Ing, Eds., pp. 23-53, Lippincott Williams \& Wilkins, Philadelphia, PA, USA, 4th edition, 2007.

[20] H. E. Fieghen, J. O. Friedrich, K. E. Burns et al., "The hemodynamic tolerability and feasibility of sustained low efficiency dialysis in the management of critically ill patients with acute kidney injury," BMC Nephrology, vol. 11, no. 1, article 32, 2010.

[21] D. Ponce, J. M. Abrão, B. B. Albino, A. L. Balbi, and J. I. Salluh, "Extended daily dialysis in acute kidney injury patients: metabolic and fluid control and risk factors for death," PLoS ONE, vol. 8, no. 12, 2013.

[22] B. B. Albino, A. L. Balbi, J. M. G. Abrão, and D. Ponce, "Dialysis complications in acute kidney injury patients treated with prolonged intermittent renal replacement therapy sessions lasting 10 versus 6 hours: results of a randomized clinical trial," Artificial Organs, vol. 39, no. 5, pp. 423-431, 2015.

[23] M. R. Marshall, T. A. Golper, M. J. Shaver, M. G. Alam, and D. K. Chatoth, "Sustained low-efficiency dialysis for critically ill patients requiring renal replacement therapy," Kidney International, vol. 60, no. 2, pp. 777-785, 2001.

[24] P. M. Palevsky, J. H. Zhang, T. Z. O'Connor et al., "Intensity of renal support in critically ill patients with acute kidney injury," The New England Journal of Medicine, vol. 359, no. 1, pp. 7-20, 2008.

[25] R. Bellomo, A. Cass, L. Cole et al., "Intensity of continuous renal-replacement therapy in critically ill patients," The New England Journal of Medicine, vol. 361, no. 17, pp. 1627-1638, 2009.

[26] J. A. Silversides, R. Pinto, R. Kuint et al., "Fluid balance, intradialytic hypotension, and outcomes in critically ill patients undergoing renal replacement therapy: a cohort study," Critical Care, vol. 18, no. 6, pp. 624-628, 2014.

[27] V. T. Costa e Silva, F. Liaño, A. Muriel, R. Díez, I. de Castro, and L. Yu, "Nephrology referral and outcomes in critically Ill acute kidney injury patients," PLoS ONE, vol. 8, no. 8, 2013.

[28] E. Q. Lima, D. M. T. Zanetta, I. Castro, and L. Yu, "Mortality risk factors and validation of severity scoring systems in critically ill patients with acute renal failure," Renal Failure, vol. 27, no. 5, pp. 547-556, 2005.

[29] J. George, S. Varma, S. Kumar, J. Thomas, S. Gopi, and R. Pisharody, "Comparing continuous venovenous hemodiafiltration and peritoneal dialysis in critically ill patients with acute kidney injury: a pilot study," Peritoneal Dialysis International, vol. 31, no. 4, pp. 422-429, 2011.

[30] D. Payen, A. C. de Pont, Y. Sakr, C. Spies, K. Reinhart, and J. L. Vincent, "A positive fluid balance is associated with a worse outcome in patients with acute renal failure," Critical Care, vol. 12, no. 3, article R74, 2008.

[31] D. Ponce, M. B. Buffarah, C. Goes, and A. Balbi, "Peritoneal dialysis in acute kidney injury: Trends in the outcome across time periods," PLoS ONE, vol. 10, no. 5, 2015. 
[32] A. Hamzić-Mehmedbašić, S. Rašić, M. Balavac, D. Rebić, M. Delić-Šarac, and A. Durak-Nalbantić, "Prognostic indicators of adverse renal outcome and death in acute kidney injury hospital survivors," Journal of Renal Injury Prevention, vol. 5, no. 2, pp. 61-68, 2016.

[33] B. B. Albino, A. L. Balbi, and A. Ponce, "Avaliação da mortalidade e recuperação da função renal de pacientes sépticos com lesão renal aguda submetidos a diferentes durações de hemodiálise prolongada," https://repositorio.unesp.br/handle/ $11449 / 151845$. 


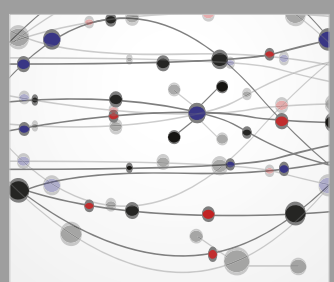

The Scientific World Journal
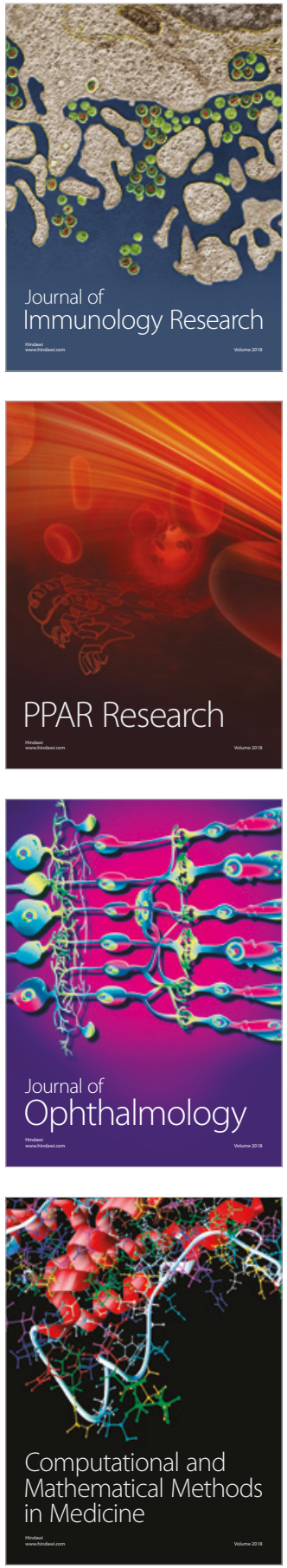

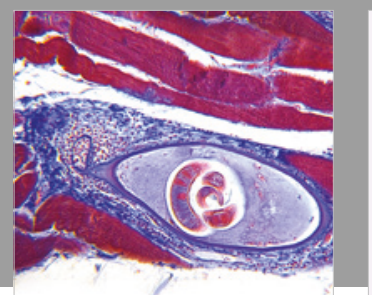

Gastroenterology Research and Practice

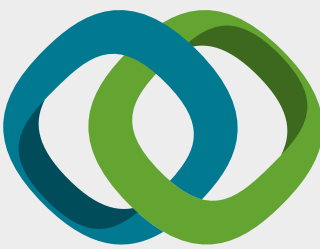

\section{Hindawi}

Submit your manuscripts at

www.hindawi.com
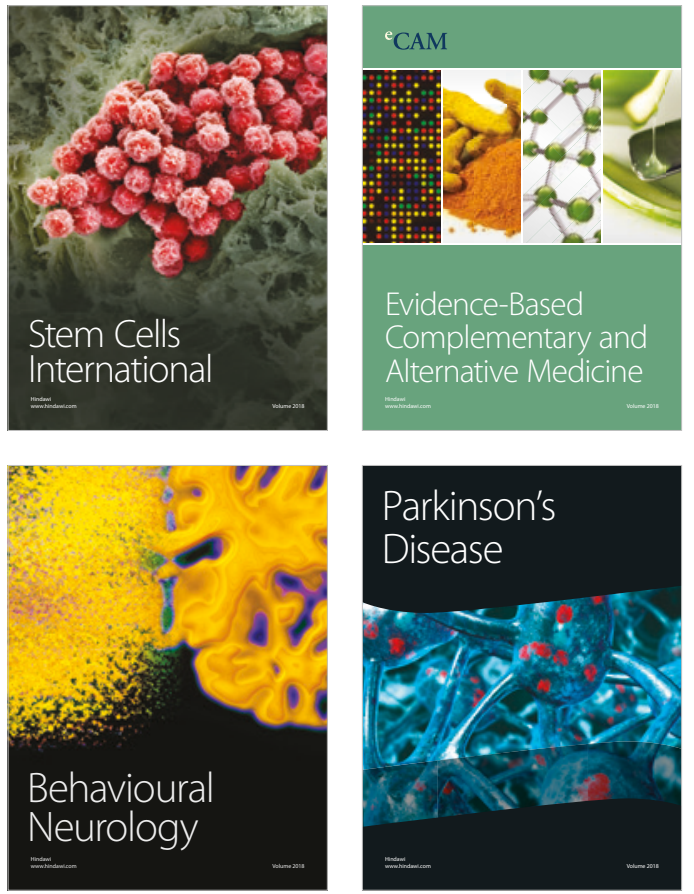

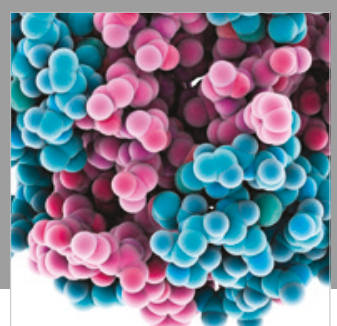

ournal of

Diabetes Research

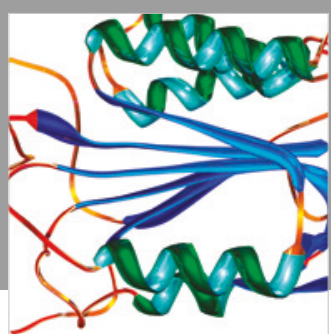

Disease Markers
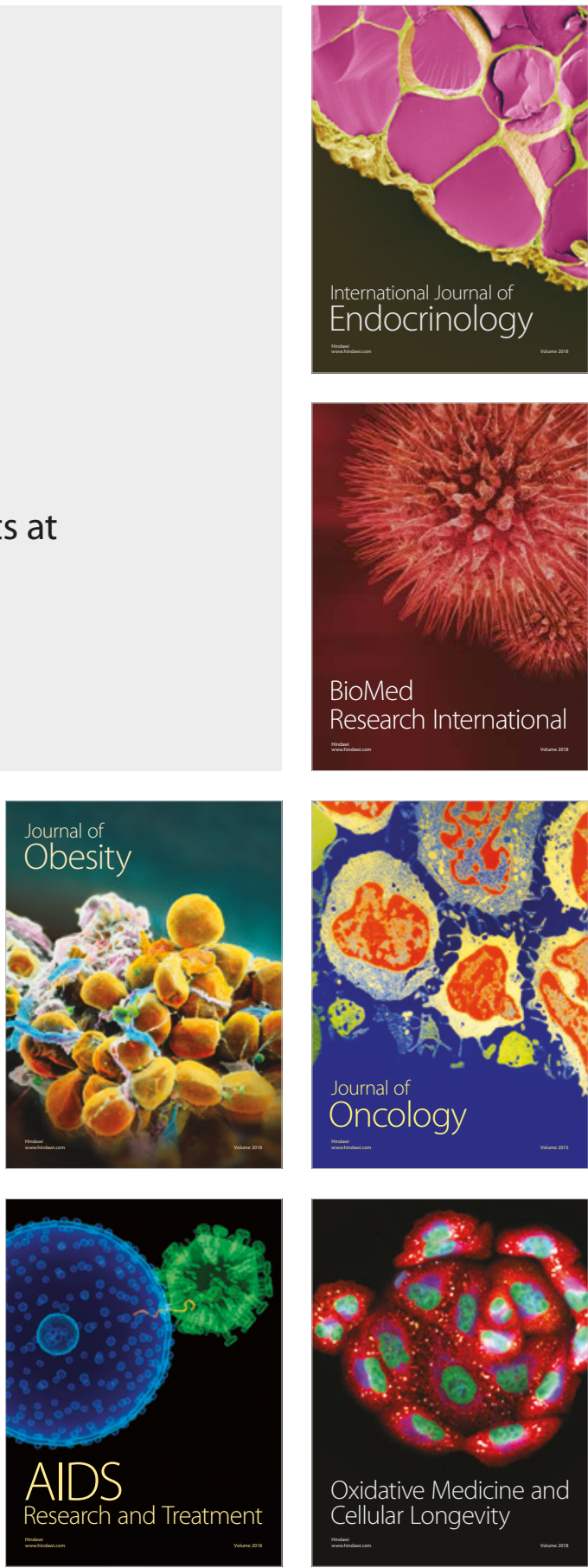